\title{
Evidence of TB Diagnostic Services at Primary Healthcare Level During COVID-19: A Scoping Review Protocol
}

Thobeka Nomzamo Dlangalala ( $\sim$ u10225120@tuks.co.za )

University of Pretoria https://orcid.org/0000-0003-1331-4247

Kuhlula Maluleke

University of Pretoria

Alfred Musekiwa

University of Pretoria

Kabelo Kgarosi

University of Pretoria

Tivani Mashamba-Thompson

University of Pretoria School of Health Systems and Public Health

\section{Protocol}

Keywords: tuberculosis, antibiotic resistant strains, Scoping Review, pandemic

Posted Date: June 16th, 2021

DOl: https://doi.org/10.21203/rs.3.rs-614440/v1

License: (c) (i) This work is licensed under a Creative Commons Attribution 4.0 International License. Read Full License 


\section{Abstract}

\section{Background}

Uninterrupted delivery of tuberculosis (TB) diagnostic services is paramount, since early diagnosis allows for timeous treatment which improves treatment outcomes, reduces the period of infectiousness and limits the development of antibiotic resistant strains of TB. In 2020, the urgency of the public health response to the COVID-19 pandemic supposedly strained many existing public health initiatives. We propose a scoping review to map the evidence of the impact of the COVID-19 pandemic on TB diagnostic services at primary healthcare $(\mathrm{PHC})$ level.

\section{Method and Analysis}

This scoping review was guided by the Arksey and O'Malley framework. We will search the databases: PubMed, Web of Science, MEDLINE Ovid, Medline EBSCO, Psyclnfo, CINAHL and Scopus. We will also search for reports from national health websites and the World Health Organization (WHO). Two independent reviewers will screen titles, abstracts and full texts of retrieved articles. The chosen articles will be represented in a data charting table. We will conduct a content thematic analysis using NVivo 10 software to extract relevant themes from the included articles. Findings will be presented according to the Preferred Reporting Items for Systematic Reviews and Meta-Analysis: Extension for Scoping Review (PRISMA-ScR). We will assess the quality of the included articles using the mixed methods assessment tool (MMAT).

\section{Discussion}

We anticipate that this scoping review will reveal the extent to which TB diagnostic services have been either maintained or disrupted by the COVID-19 pandemic. This information will direct future research, and reveal barriers and enablers for TB diagnostic services during a pandemic.

\section{Introduction}

Despite being preventable and curable, 10 million people contracted tuberculosis (TB) in 2019 [1]. During the same period, 2.9 million people who contracted TB were missed by national notification systems [2]. These missing individuals contribute to continued transmission [3], while long diagnostic delays exacerbate disease severity and ongoing spread [4]. Reaching these missing people through rapid TB diagnostic services, preferably at primary healthcare level (PHC), can help to end the TB epidemic [2, 5]. In 2020, COVID-19 emerged, hindering global TB control efforts [6], particularly screening and diagnostics services $[7,8]$. These services suffered a sharp decline due to lockdowns limiting access to healthcare and a rise in fear and stigma since the advent of COVID-19 $[7,9,10]$.

Timely diagnoses and treatment is a major facet of TB control, which is one of the aims of the World Health Organization's (WHO) End TB strategy [11]. While the strategy aims to eliminate the TB epidemic 
by 2035 , the WHO has set interim milestones to reduce TB deaths and TB incidence rates by 2020 and $2025[2,11]$. The 2020 TB global health report shows that 2020 milestones were not met [2]. Similarly, the United Nations (UN) set targets to diagnose and treat 40 million additional people by 2022 [6]. Although progress is being made, the current numbers suggest that ending TB by 2035 is unattainable [2, 12]. It is possible that the small gains made toward controlling TB were disrupted by the COVID-19 pandemic, pushing the 2035 goals further into the future $[6,13]$.

To ensure that these targets will be achieved, TB diagnostic services should not be interrupted, even when there are other public health priorities, such as the COVID-19 pandemic. The evidence for the impact of the COVID-19 pandemic on TB diagnostic services at the PHC level remains unclear. We propose to map evidence on TB diagnostic services at PHC level during the COVID-19 pandemic. We anticipate that our results will provide valuable evidence to guide future research and reveal areas that need to be addressed to improve TB diagnostic services at PHC level during the COVID-19 pandemic and aid global efforts to end TB.

\section{Methods}

\section{Protocol Design}

We will conduct a scoping review of the available evidence on TB diagnostic services during the COVID19 era. The proposed scoping review will be guided by the Arksey and O'Malley framework [14], Levac et al [15], and the Joanna Briggs Institute 2020 guidelines [16]. The Arksey and O'Malley framework suggests that scoping reviews should be conducted in the following five steps:

Step 1: Identifying the research question

Step 2: Identifying relevant studies

Step 3: Selecting studies

Step 3: Charting the data

Step 4: Collating, summarizing and reporting the data

The results of the review will be reported according to the guidelines for the Preferred Reporting Items for Systematic Reviews and Meta-analysis: Extension for Scoping Reviews (PRISMA-ScR) [17].

\section{Step 1: Identifying the research question}

The primary research question is: What is the evidence on TB diagnostic services at PHC level during the COVID-19 pandemic?

We will assess the eligibility of the research questions using the Population, Concept and Context (PCC) nomenclature developed by the Joanna Briggs Institute [16]. The PCC statement will guide the selection 
of studies (Table 1).

\section{Step 2: Identifying relevant studies}

We will conduct an advanced search in the following electronic databases: PubMed, Web of Science, MEDLINE Ovid, Medline EBSCO, PsycInfo, CINAHL and Scopus. We will also retrieve relevant literature from the WHO website and national health websites. We will manually search the reference lists of all included studies to identify additional literature. Studies will be identified using the following keywords and Medical Subject Heading (MeSH) terms; "TB diagnostics", "TB testing" "COVID-19", "SARS-CoV-2", "COVID-19 Pandemic", "COVID-19 era" and "Primary healthcare". A librarian will ensure that the best search strategies are used for each database, to ensure the best results. We conducted a pilot search in PubMed to determine the feasibility of the proposed scoping review (Table 2).

We will use a search summary table to ensure transparency, reliability and reproducibility of our search (Table 3). This table will also record the effectiveness of our strategy for each database in terms of sensitivity, precision, recall, yield, number needed to read (NNR) and number of unique records [18]. We will rerun searches in databases that yielded the most relevant articles to find articles that might have been missed during the original search [18].

\section{Inclusion criteria}

Studies will be included if they:

- report the effects of COVID-19 on TB diagnostics services

- focus on COVID-19 and TB diagnostics services

- report on COVID-19 and TB diagnostics from PHC

\section{Exclusion criteria}

Studies will be excluded if they:

- report on viral outbreaks other than SARS-CoV-2

- report on TB services outside PHC

- report on the effects of COVID-19 on other diseases and conditions beside TB

- Articles from before December 2019

\section{Step 3: Selecting studies}

After retrieving relevant articles, the principal investigator will screen the titles of each article. Eligible articles will be uploaded to a library in EndNote 20 where duplicates will be identified and deleted. Two independent reviewers will screen abstracts of the included articles using a screening tool that specifies inclusion and exclusion criteria. The independent reviewers will discuss any discrepancies arising from the abstract screening stage until they agree on the selected articles. Using the screening tool, the two 
reviewers will then screen the full-texts of the relevant articles. Any discrepancies arising after full text screening will be resolved by a third reviewer. A librarian will assist with retrieving inaccessible articles. A PRISMA-ScR flow diagram will then be used to report results [17].

\section{Step 4: Charting the data}

An electronic data charting form will be used to capture the relevant data from each selected article.

\section{Quality appraisal}

The quality of included studies will be assessed using the Mixed Methods Appraisal Tool (MMAT) V.2018 software [19]. We will assess the methodological quality of included qualitative, quantitative and mixed methods studies. The MMAT tool ranks studies as low quality (score $\leq 50 \%$ ), average quality (score $\geq$ $51-75 \%$ ) and high quality (score $\geq 76-100 \%$ ). Appraising the quality of studies will ensure that we identify and understand the strength, weaknesses and potential bias of studies.

\section{Step 5: Collating, summarizing and reporting results}

We will conduct a content thematic analysis, using NVivo 10 software, to extract data and summarize findings [20]. We will summarize the results using a narrative approach. The reviewers will meticulously analyze the emerging themes from the included articles in relation to the research question. The findings of this scoping review will identify the influence of the COVID-19 pandemic on TB diagnostics at PHC level.

\section{Discussion}

The proposed scoping review will map existing global evidence on TB diagnostic services during the COVID-19 pandemic. The WHO has set targets to reduce TB deaths and incidence rates by $90 \%$ and $80 \%$ of 2015 values, respectively, by 2030 [1]. Ultimately, the WHO aims to end TB by 2035. The end TB strategy centers around three pillars, the first of which focuses on timely diagnosis of patients including universal drug susceptibility testing [12]. Ensuring that diagnostic services are accessible at PHC level will guarantee that treatment and care are available to all members of society who need them.

This review forms part of a large research project investigating the effect of COVID-19 on TB diagnostic services in primary health care clinics in Kwa-Zulu Natal, South Africa. As such, we will focus on evidence from PHC level facilities and will be limited to articles that present evidence of TB diagnostic services during the COVID-19 pandemic. For the same reason evidence on service disruptions of other diseases during COVID-19 will be excluded. Since COVID-19 officially emerged in December 2019 studies published before December 2019, will not be included. Additionally, we will not include reports from other viral epidemics because the review is focused on TB services in the COVID-19 era.

In conclusion, we anticipate that the proposed scoping review will uncover the effects of the COVID-19 pandemic on TB diagnostic services across the globe. This review will also highlight knowledge gaps in 
this area which will help stimulate research that could improve TB diagnostic services at PHC level in line with the WHO's goal to eliminate TB.

\section{Abbreviations}

MeSH: $\quad$ Medical Subject Headings

MMAT: $\quad$ Mixed Methods Appraisal Tool

PHC: $\quad$ Primary Healthcare

PRISMA-ScR: Preferred Reporting Items for Systematic Reviews and Meta-Analyses Extension for Scoping Reviews

TB: Tuberculosis

UN: United Nations

WHO: World Health Organization

\section{Declarations}

\section{Availability of data and materials}

The data generated and analysed from this study will be presented and published in a systematic scoping review article.

\section{Acknowledgements}

Faculty of Health Sciences, University of Pretoria, South Africa. Thank you to Dr. Cheryl Tosh for editing.

\section{Funding}

The authors would like to thank NinetyOne SA (PTY) LTD for covering tuition and living expenses of the primary investigator during her PhD.

\section{Author Information}

Affiliations

School of Health Systems and Public Health, Faculty of Health Science, University of Pretoria, Pretoria, South Africa

Thobeka Dlangalala

Kuhlala Mululeke 
Tivani Mashamba-Thompson

Alfred Musekiwa

Library services, Faculty of Health Sciences, University of Pretoria, South Africa

Kabelo Kgarosi

Contributions

TD prepared the original draft and conceptualized the study under the supervision of TM-T and AM. KM critically reviewed the final draft. KK helped to develop and refine the search strategy. AM for critically reviewed final draft and approved final manuscript. TM-T reviewed all drafts and approved final manuscript.

Corresponding Author

Thobeka Dlangalala

\section{Ethical Declarations}

Ethics approval and consent to participate

No humans or animals were used to conduct this study. All data was obtained from published data literature.

Consent for Publication

Not Applicable

Competing interests

The authors have no conflicts of interests to declare

\section{References}

1. Tuberculosis [https://www.who.int/news-room/fact-sheets/detail/tuberculosis]

2. Chakaya J, Khan M, Ntoumi F, Aklillu E, Fatima R, Mwaba P, Kapata N, Mfinanga S, Hasnain SE, Katoto PDMC et al: Global Tuberculosis Report 2020 - Reflections on the Global TB burden, treatment and prevention efforts. International Journal of Infectious Diseases 2021, 0.

3. Pai M, Nicol MP, Boehme CC: Tuberculosis Diagnostics: State of the Art and Future Directions. Microbiology Spectrum 2016, 4:1-15. 
4. Nathavitharana RR: Stamping out tuberculosis: The importance of diagnostic innovation and effective implementation. Annals of the American Thoracic Society 2019, 16:1112-1113.

5. Dheda K, Ruhwald M, Theron G, Peter J, Yam WC: Point-of-care diagnosis of tuberculosis: Past, present and future. Respirology 2013, 18:217-232.

6. Sahu S, Ditiu L, Sachdeva KS, Zumla A: Recovering from the Impact of the Covid-19 Pandemic and Accelerating to Achieving the United Nations General Assembly Tuberculosis Targets. International Journal of Infectious Diseases 2021.

7. Alene KA, Wangdi K, Clements ACA: Impact of the COVID-19 Pandemic on Tuberculosis Control: An Overview. Tropical Medicine and Infectious Disease 2020, 5:123.

8. Fei H, Yinyin X, Hui C, Ni W, Xin D, Wei C, Tao L, Shitong H, Miaomiao S, Mingting C et al: The impact of the COVID-19 epidemic on tuberculosis control in China. The Lancet Regional Health - Western Pacific 2020, 3:100032.

9. Karim QA, Karim SSA: COVID-19 affects HIV and tuberculosis care. Science 2020, 369:366-368.

10. Jain VK, lyengar KP, Samy DA, Vaishya R: Tuberculosis in the era of COVID-19 in India. Diabetes and Metabolic Syndrome: Clinical Research and Reviews 2020, 14:1439-1443.

11. Uplekar M, Weil D, Lonnroth K, Jaramillo E, Lienhardt C, Dias HM, Falzon D, Floyd K, Gargioni G, Getahun H et al: WHO's new end TB strategy. The Lancet 2015, 385:1799-1801.

12. End TB Strategy [https://www.who.int/tb/End_TB_brochure.pdf?ua=1]

13. Zumla A, Chakaya J, Khan M, Fatima R, Wejse C, Al-Abri S, Fox GJ, Nachega J, Kapata N, Knipper $M$ et al: World Tuberculosis Day 2021 Theme - 'THE CLOCK IS TICKING' - and the world is running out of time to deliver the United Nations General Assembly commitments to End TB due to the COVID-19 pandemic. International Journal of Infectious Diseases 2021.

14. Arksey H, O'Malley L: Scoping studies: Towards a methodological framework. International Journal of Social Research Methodology: Theory and Practice 2005, 8:19-32.

15. Levac D, Colquhoun H, O'Brien KK: Scoping studies: Advancing the methodology. Implementation Science 2010, 5:69.

16. Peters MD, et al.: Updated methodological guidance for the conduct of scoping reviews. JBI Evidence Synthesis 2020, 18(10):2119.

17. Tricco AC, Lillie E, Zarin W, O'Brien KK, Colquhoun H, Levac D, Moher D, Peters MDJ, Horsley T, Weeks $L$ et al: PRISMA extension for scoping reviews (PRISMA-ScR): Checklist and explanation. In: Annals of Internal Medicine. vol. 169: American College of Physicians; 2018: 467-473. 
18. Bethel AC, Rogers $M$, Abbott $R$ : Use of a search summary table to improve systematic review search methods, results, and efficiency. Journal of the Medical Library Association 2021, 109(1).

19. Nha HONG Q, Pluye P, Fàbregues S, Bartlett G, Boardman F, Cargo M, Dagenais P, Gagnon M-P, Griffiths F, Nicolau B et al: MIXED METHODS APPRAISAL TOOL (MMAT) VERSION 2018 User guide. In.

20. Braun V, Clarke V: Using thematic analysis in psychology. Qualitative Research in Psychology 2006, 3:77-101.

\section{Supplementary Files}

This is a list of supplementary files associated with this preprint. Click to download.

- PRISMAPchecklist.docx 